\title{
Piroplasmose du porc \\ Infection naturelle à Piroplasma trautmanni Knut et du Toit, 1921, à Bambari (République Centrafricaine)
}

\author{
por J. ITARD
}

Instılut d'Elevage et de Médecine Vétérınaire des Pays tropicaux

(Laboratoire d'entomo-protozoologie)

\begin{abstract}
RÉSUMÉ
A Bambari (République centrafricaıne) chez une truse soignée pour une métrite, il a été observé une infestation à Piroplosma trautmanni. Les tiques récollées appartiennent à l'espèce Rhıpicephalus senegolensis. L'auteur, après une étude morphologique du parasite, présente une série de microphotographies des différentes formes parasitaires.
\end{abstract}

La.piroplasmose du porc est une affection assez rarement observée et n'ayant fait l'objet que d'un nombre relativement restreint de publications.

Signalée pour la première fois en 1911 en Russie, puis selon KNUTH ef DU TOIT, par TRAUTMANN au Tanganyika en 1914, la Piroplasmose du porc fut particulièrement bien étudiée par les auteurs italiens (SPARAPANI, 1917, LANZILLO, 1924 et 1933 ; CERRUTI, 1934 et 1939).

Elle fut observée par la suite en Guinée française en 1942, en Bulgarie (1943 et 1946), au Congo Belge et au Transvaal (1948), en Rhodésie du Sud par LAWRENCE et SHONE en 1948 et 1955 ef en Guinée portugaise par TElNDERO en 1952. En 1960, enfin SHONE ef PHILIP montrent que le potamochère (Potomochoerus porcus mochona) peut héberger le parasite bien que l'infection soit inapparente chez ce suidé.

It ne semble pas, à notre connassance tout au moins, que cette maladie alt fait l'objet d'autres publications. En ce qui concerne plus particulièrement les territoires africains d'expression française, les observations concernant la piro- plasmose du porc sont extrêmement réduites (*).

C'est pourquoi, ayant eu l'occasion de diagnostiquer, en avril 1962, une infection à Piroplasma troutmanni, chez une truie metis Largewhite appartenant à un commerçant de Bambari (République centrafricaıne), nous avons pensé qu'il pourrait être intéressant d'exposer $\mathrm{ICl}$ cette observation.

L'animal infecté était soigné pour une métrite mais l'infirmier vétérinaire chargé du traitement ayant constaté un léger ictère, effectua des étalements de sang, qui nous furent soumis pour examen.

Après coloration des étalements au MayGrunwald-Giemsa, l'examen microscopıque mit en évidence de nombreuses hématies parasitées par un protozoaire que, d'après sa structure, sa forme et ses dimensions, nous avons identifié à Piroplasmo trautmanni.

(*) Signalons ici que la Babesiellose du porc, à Babesiella perroncitoi a été observée au Soudan, en 1942, par ROUSSELOT (Notes de Parasitologie 1ropicale) et en R. C. A., en 1959, par FINELLE et MARTIN (rapport annuel du Laboratoire de Farcha (Tehad), 1959), 


\section{SYMPTÔMES}

Cliniquement, l'affection ne s'est caractérisée par aucun symptôme précis, si ce n'est un léger ictère. Pas d'anémie (l'examen des étalements de sang ne révèle pas davantage de lésions érythrocytaires); pas d'hémoglobinurie ; température légèrement supérieure à la normale; métrite. Tels sont les seuls signes clinıques que nous ayons pu relever. On peut se demander si la métrite est une conséquence de l'infestation parasitaire ou si celle-ci, au contraire, n'est apparve qu'à la faveur d'une diminution de la résistance de l'animal, provoquée par l'infection microbienne. LAWRENCE et SHONE notent cependant que l'avortement, en Rhodésie du Sud, est un sympiôme fréquent de la Piroplasmose du porc. TEINDERO a également noté un cas d'avortement chez une truie parasitée par Piroplasma trautmanni, en Guinée portugaise.

\section{TRANSMISSION}

On a longtemps attribué la transmission de l'affection, en Afrique, à Boophrlus decolorotus, mais aucune recherche expérimentale n'ayant été réalisée, le rôle vecteur de cette espèce reste hypothétique. II semble qu'il faille plutôt attribuer ce rôle vecteur aux Rhipicephalus. TEINDERO, en effet, en Guinée portugaise, a récolté sur des porcs infectés Rhipicephalus lunulatus. SHONE et PHILIP, en Rhodésie, ont récolté plusieurs Rhipicephalus simus et un Rhipicephalus appendiculatus. FINELLE et MARTIN (1959), sur des porcs atteints de Babesiellose à Babesiella perroncitoi, à Bangui, ont récolté Rhipicepholus complanotus, parasite typique du potamochère en forêt équatoriale occidentale.

Nous avons nous-même trouvé sur le corps de l'animal objet de la présente observation, quelques Rhipicephalus senegalensis (MOREL détermınateur) et un grand nombre d'Hematopinus suis.

\section{TRAITEMENT}

L'animal parasıté, outre le traitement classıque des métrites, reçut une injection unique par voie sous-cutanée, de Zothélone. Tout rentra très rapidement dans l'ordre, ef l'animal guérit en quelques jours de son infection microbienne comme de sa piroplasmose. La stéritisation parasitaire fut constatée au bout de $48 \mathrm{~h}$, dans les étalements de sang.

\section{MORPHOLOGIE DU PARASITE (*)}

L'infestation parasitaire est en moyenne de 3 hématies parasitées pour 1.000 .

Le pourcentage des diverses formes paras।taires, classées selon les groupes décrits par les chercheurs de l'institut Pasteur d'Algérie, a été établi après comptage de 500 parasites. Nous avons dans chaque cas, calculé l'intervalle de confiance à 5 p. 100, selon la formule:

$$
p=p o \pm 2 \sqrt{\frac{p_{0} q_{0}}{n}}
$$

où $p$ représente le pourcentage théorique: $p_{0}$, le pourcentage observé; $q_{0}=1-p_{0} ; n=500$.

Nous indiquons, dans le tableau $l$, le nombre de formes parasitaires, le pourcentage observé et les lımites inférieures et supérieures du pourcentage théorique, avec un risque d'erreur de 5 p. 100.

II nous a paru intéressant de comparer ces pourcentages avec ceux observés par TEINDERO en 1951.

Le tableau II Indique les proportions des formes parasitaıres relevées par TEINDERO au cours de deux examens successifs et en regard celles que nous avons notées à Bambari. Ces pourcentages sont pratiquement identiques.

Les parasites prennent en falt les aspects les plus divers et lorsqu'une hématie en contient plusieurs, ils peuvent occuper à l'intérieur du globule rouge, les positions les plus variables.

Il est souvent difficile de faire rentrer un parasite dans une catégorie ou dans l'autre, les stades intermédıarres étant fréquents. Les formes libres sont nombreuses; elles ont généralement un aspect piriforme, plus ou moins allongé ( $\mathrm{Pl} . \mathrm{IV}$, Fig. 22 et $23 ; \mathrm{Pl}$. V, Fig. 25). On rencontre parfois des formes rondes libres. (PI. IV, Fig. 24).

$\left(^{*}\right)$ Cette étude fut effectuée, en 1964, au laboratoire d'entomo-protozoologie de l'l. E. M. V. T. à MaisonsAlfort. Les microphotographies que nous présentons (grossissement $=2.000$ environ) ont été réalisées par le DrGAYOT, chargé de Recherche au laboratoire central de recherches vétérinaires à Maisons-Alfort, auquel nous adressons tous nos remerciements pour l'aide considérable qu'il nous a apportée. 
TABLEAU NO I

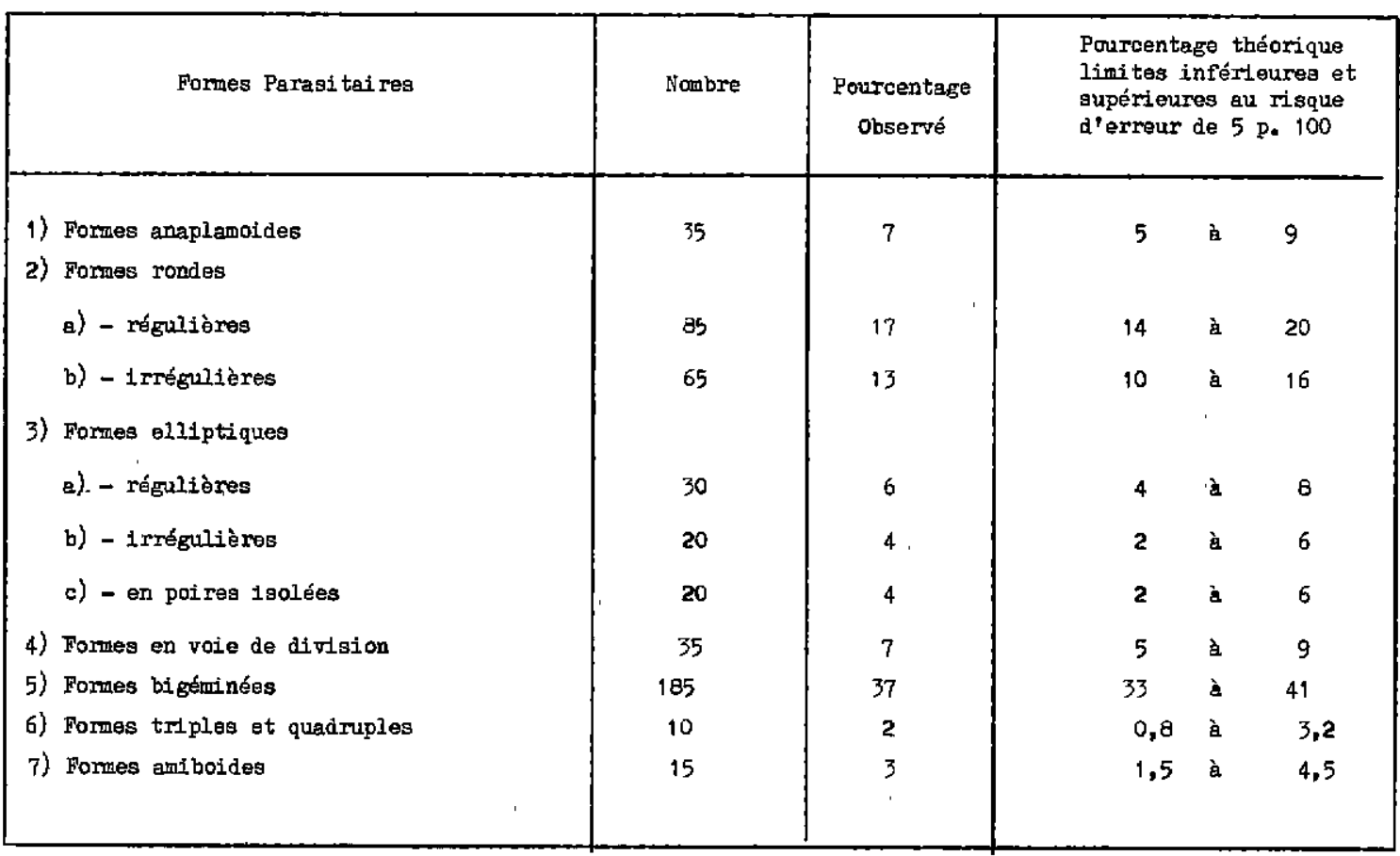

TABLASO YT II

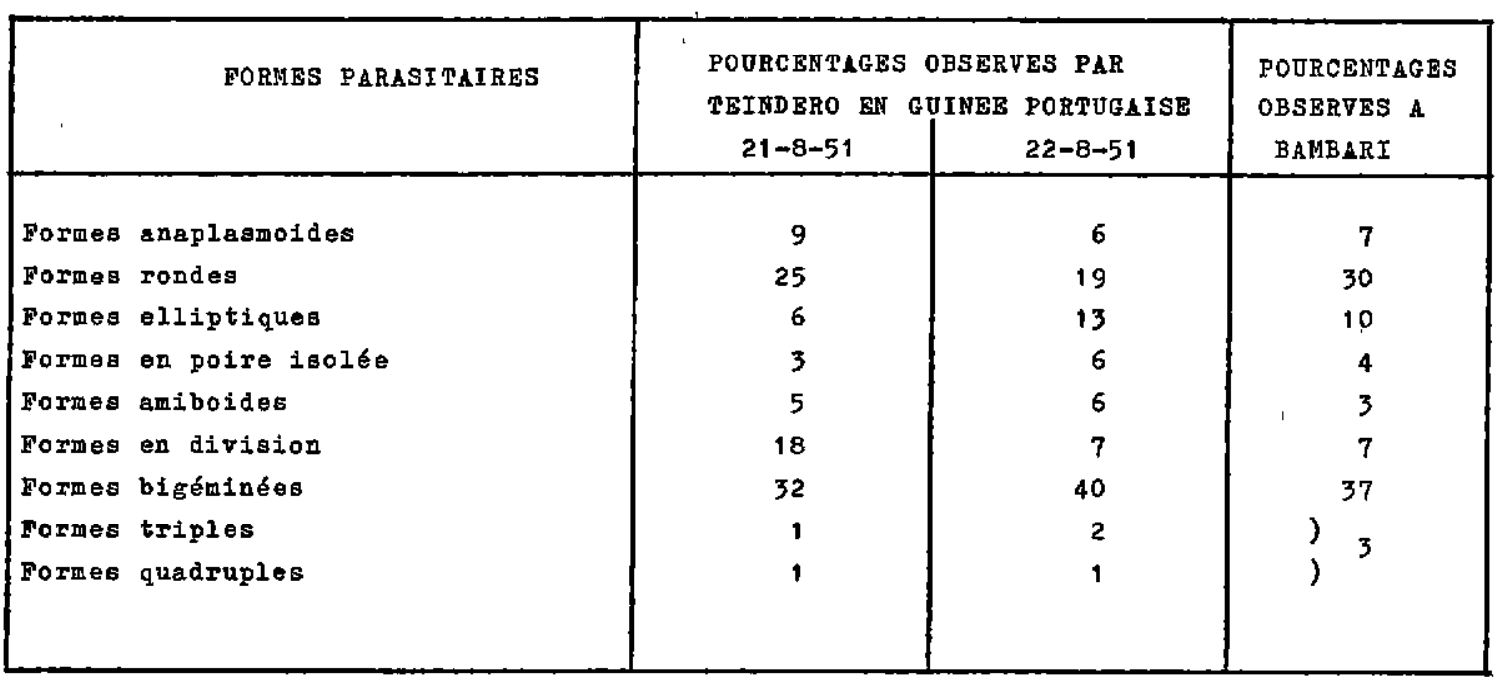


Formes anaplasmoïdes : Elles se présentent sous la forme de gros grains intraglobulaires. colorés en violet pourpre et d'un diamètre moyen de $1,2 \mu$.

On note parfois, partant du granule de chromatine, de fins prolongements cytoplasmıques, se colorant mal et dont la forme et les dimensions sont très variables (PI. II, Fig. 6).

Formes rondes (PI. II, Fig. 4; Pl. IV, Fig. 13 et 14). Uniques dans un globule rouge, elles peuvent être plus ou moins grandes (diamètre compris entre $2 \mu$ et $3,5 \mu$ ), régulièrement rondes ou plus au moins ovales. Elles possèdent parfors un petit appendice qui les rapproche des formes en poires. Le cytoplasme et la chromatine sont généralement répartis à la périphérie du parasite, laissant une vacuole centrale plus ou moins grande. Des prolongements, de nature chromatique, peuvent traverser cette vacuole centrale et former à l'intérieur de celle-ci des figures complexes.

Formes elliptiques (PI. I, Fig. 1 ; PI. III, Fig. 7 et $8 ;$ Pl. IV, Fig. 15, 16 et 17).

On peut rencontrer tous les stades entre les formes rondes régulières et les formes elliptiques et la classification est souvent malaisée.

Les formes elliptiques peuvent être parfaitement régulières ou en poires isolées, ou fusiformes et plus ou moins contournées.

Le cytopiasme, plus ou moins dense, est généralement répartı sur le pourtour du parasite et laisse une vacuole centrale à contours plus ou moins nets. La chromatine est située de façon très variable : tantôt rassemblée sur un côté du parasite (Fig. 1 et 17) tantôt plus ou moins régulièrement disposée à la périphérie (Fig. 7 et 15), elle peut dans les formes en poires en particulier, être divisée en deux masses ou plus, séparées plus ou moins nettement les unes des cutres (Fig. 8 et 16).

Leurs dimensions varient de 3 à $6 \mu$ de longueur sur $1 \mu$ à $2,5 \mu$ de largeur.

Formes en voie de division (PI. I, Fig. 3 ; Pl. III, Fig. 7 et $8 ;$ PI. V, Fig. 31 et 32 ).

Les formes parfaites de division sont rares. Plus souvent on trouve deux parasites étroitement accolés avec une plage commune de cytoplasme à leur partie acumınée et une échancrure nette séparant les deux pôles mousses à l'extrémifé opposée.
Formes bigéminées (PI. I, Fig. $1 ; \mathrm{Pl}$. II, Fig. 4 ef 5 ; PI. III, Fig. 9 d 12 ; Pl. IV, Fig. 18 et 22 ;.P. , V Fig. 26 d̀ 30,33 à 36).

Ce sont les formes les plus fréquemment rencontrées. On trouve tous les stades entre la forme géminée classique, dont les éléments en poire forment entre eux un angle très aıgu, la longueur de chaque élément étant nettement supérleure au rayon de l'hématie ef les petites formes géminées presque rondes formant entre elles un angle plus ou moins ouvert pouvant parfois atteindre $180^{\circ}$ et ressemblant d̀ des Babesiella.

Les éléments piriformes peuvent être réunis par leur extrémité pointue ou être nettement séparés, s'allongeant côte à côte ou occupant à l'intérieur de l'hématie des positions très variables. Certaıns éléments sont en opposition, leurs grands axes étant parallèles. Dans d'autres cas, les grands axes sont plus ou moins perpendiculaires l'un à l'cutre. Certaines formes géminées sont dissymétriques, l'un des éléments ayant un aspect piriforme classique, l'autre étant plus petit et arrondi.

Le cytoplame est souvent percé d'une ou plusieurs vacuoles d̀ contours plus ou moins nets et la chromatine peut y occuper des positions diverses. On trouve des éléments dont la chromatine se concentre en position équatoriale ou parfois vers l'un des pôles. Dans d'autres cas, la chromatine est divisée en deux masses ou plus, répartıes irrégulièrement à la périphérıe du parasite et réunies ou non par des filaments plus au moins denses.

Formes triples ef quadruples (PI. II, Fig. 5 et 6 : PI. III, Fig. 12 ; PI. IV, Fig. 21).

Peu nombreuses, elles procèdent soit par divisions longitudinales multiples, des formes en poire, soit plus fréquemment par bourgeonnement, des formes amibordes.

Formes amiboïdes (Pl. I, Fig. 3 ; Pl. IV, Fig. 19 et 20).

Elles ont généralement une forme plus ou moins arrondie, avec des prolongements obtus assez semblables d̀ des pseudopodes.

Formes libres (PI. IV, Fig. 22 d̀ 24 ; Pl. V, Fig. 25).

Ce sont généralement des formes bigémınées lıbres côte à côte dans le plasma. Les formes libres en poire isolée ne sont cependant pas 
rares. On discerne quelquefois, au-dessous de ceritaines formes libres, les vestiges du globule rouge qui les contenat.

\section{PLANCHE}

1. - Forme elliptique et forme bigéminée.

2. - Piroplasmes phagocytés par un macrophage.

3. - Forme amiboide et forme en division.

\section{PLANCHE !I}

4 - Forme ronde et forme bıgéminée.

5. - Forme bigéminée et forme quadruple.

6. - Forme triple el forme anaplasmoide.

\section{PLANCHE II]}

7. - Forme elliptique et forme en division.

B. - Forme elliptıque ef forme en division.

9. - Forme bıgéminée.

10. - Formes bigéminées.

11. - Formes bigéminées.

12. - Formes bigéminées el forme triple.

\section{PLANCHE IV}

13. - Forme ronde.

14. - Forme ronde.

15. - Forme elliptique

16. - Forme en porre isolée.
Nous avons trouvé également des formes parasitaires phagocytées par un macrophage (PI. I, Fig. 2).
17. - Forme elliptique.

18. - Forme bigéminée.

19. - Forme amiboìde.

20. - Forme amiborde.

2h. - Forme quadruple.

22 - Forme gémınée et forme lıbre en poire.

23. - Forme libre en poire.

24. - Formes rondes libres.

\section{PLANCHE V}

25. - Formes libres en poire.

26. - Forme bigéminée.

27. - Forme bigéminée.

28. - Forme bigéminée.

29. - Forme bigéminée.

30. - Forme bıgéminée.

31. - Forme en division.

32. - Forme en division.

33. - Forme bigémınée.

34. - Forme bigémınée.

35. - Forme bigéminée.

36. - Forme bigémınée $\left(180^{\circ}\right)$.

\section{SUMMARY}

Piroplasmosis in Swine.

Natural infection with Piroplasma trautmann:

(KNUTH and DU TOIT, 1921)

in Bambari (Central African Republic)

The author has observed a case of Piroplasmosis due to Piroplas no trautmannt in a sow treated for metritis. The collected ticks balonged to the species Rhipicepholus senegalensıs. A morphological study and microphotographes are presented. 


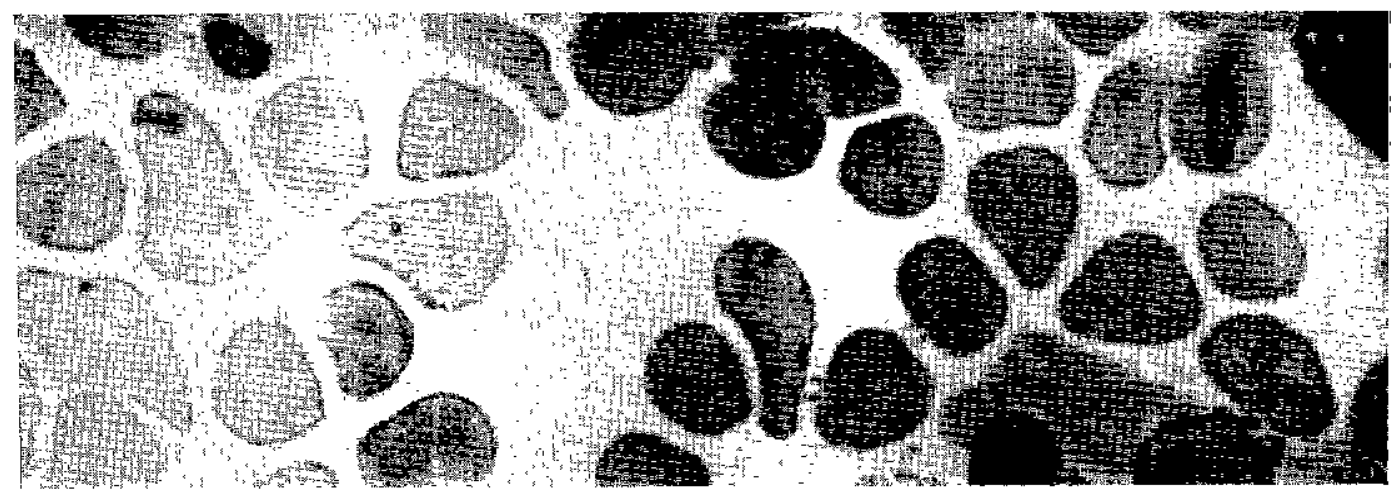

Fig. 1

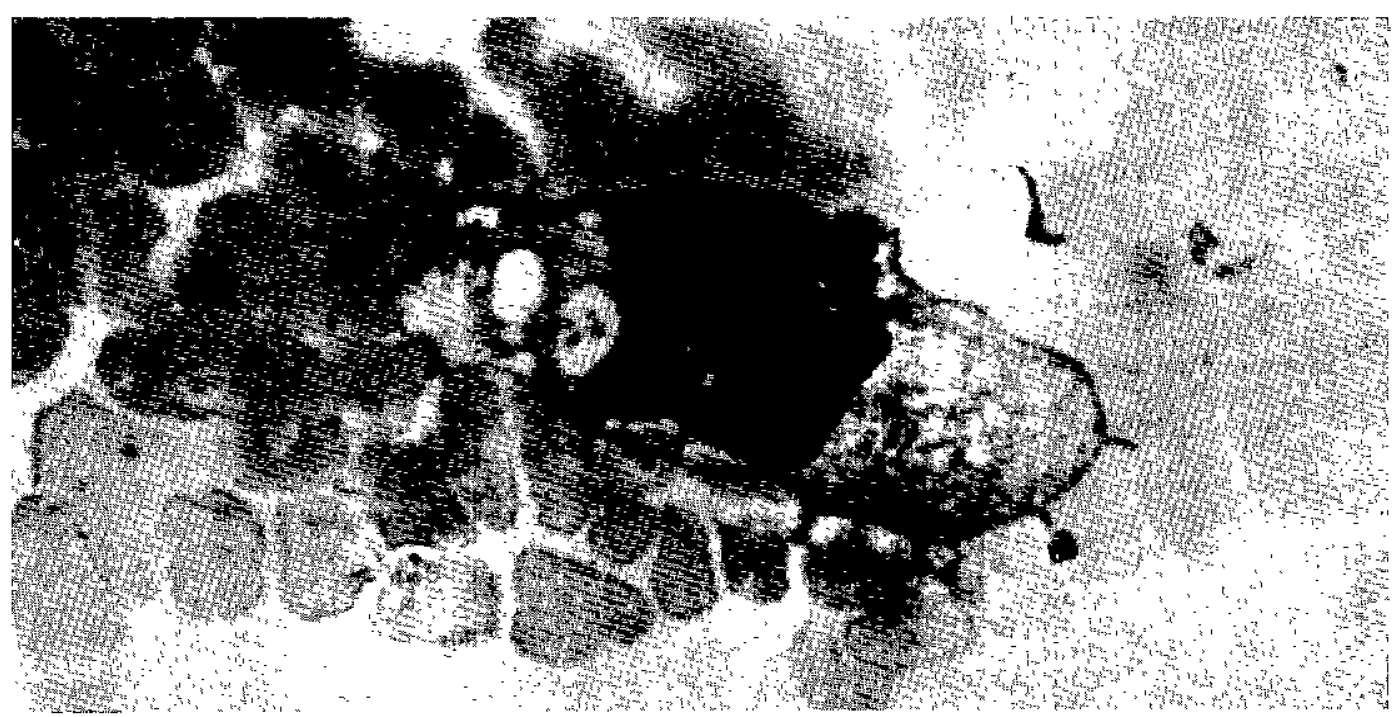

Fig. 2

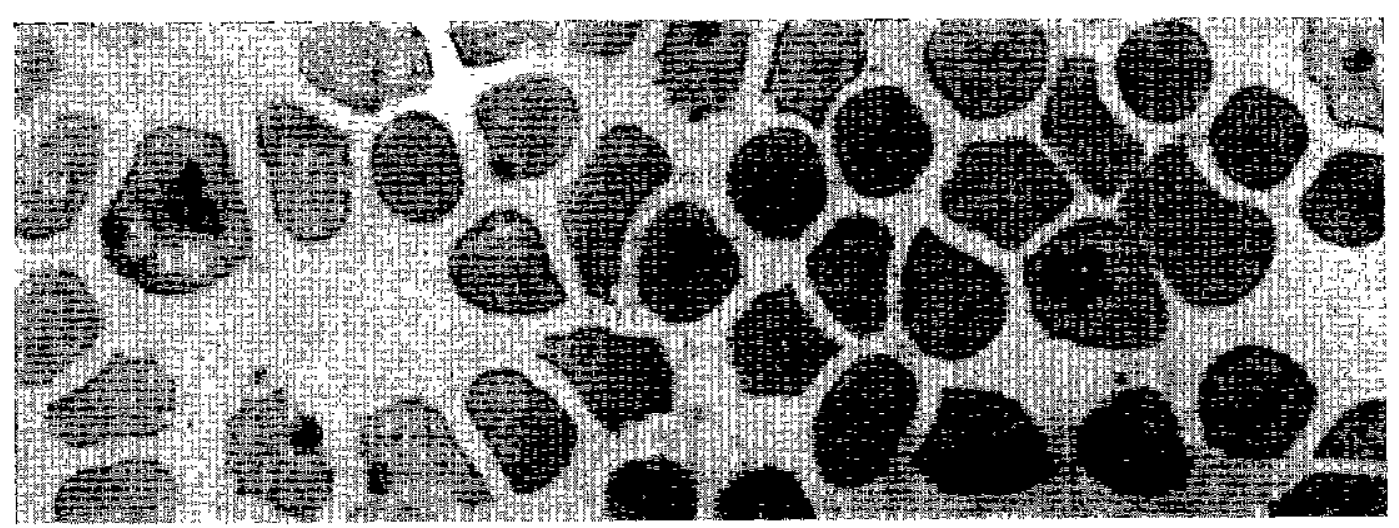

Fig. 3 


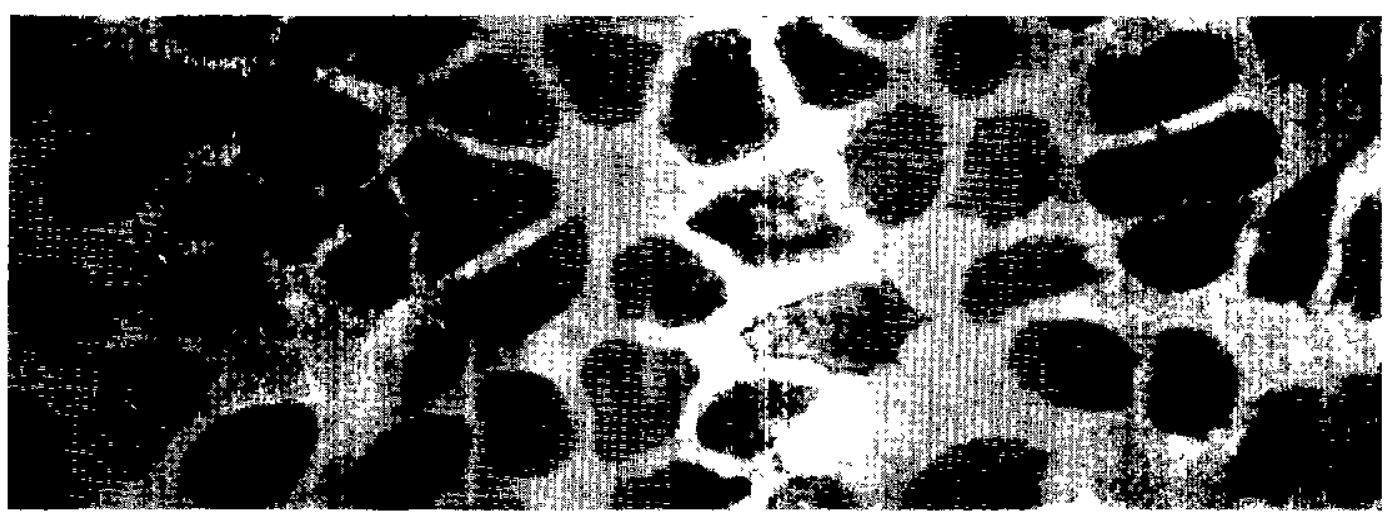

Fig. 4

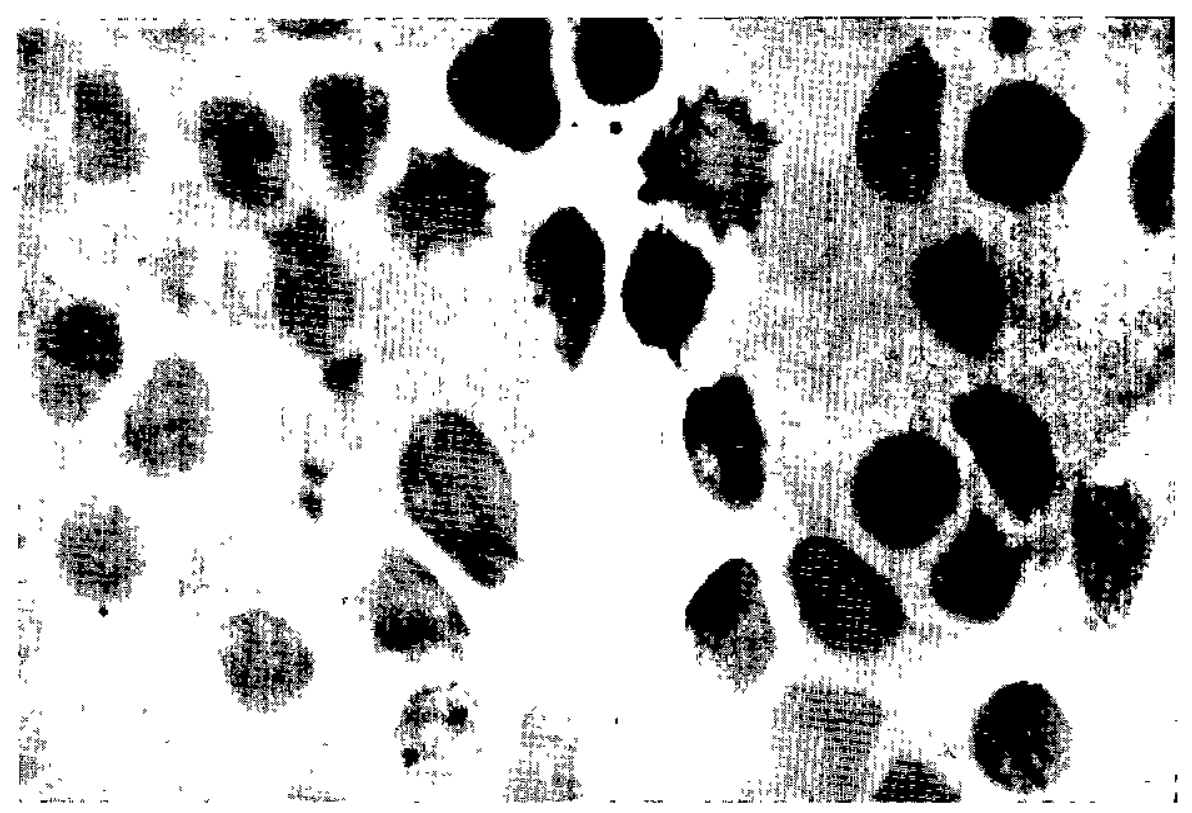

Fig. 5

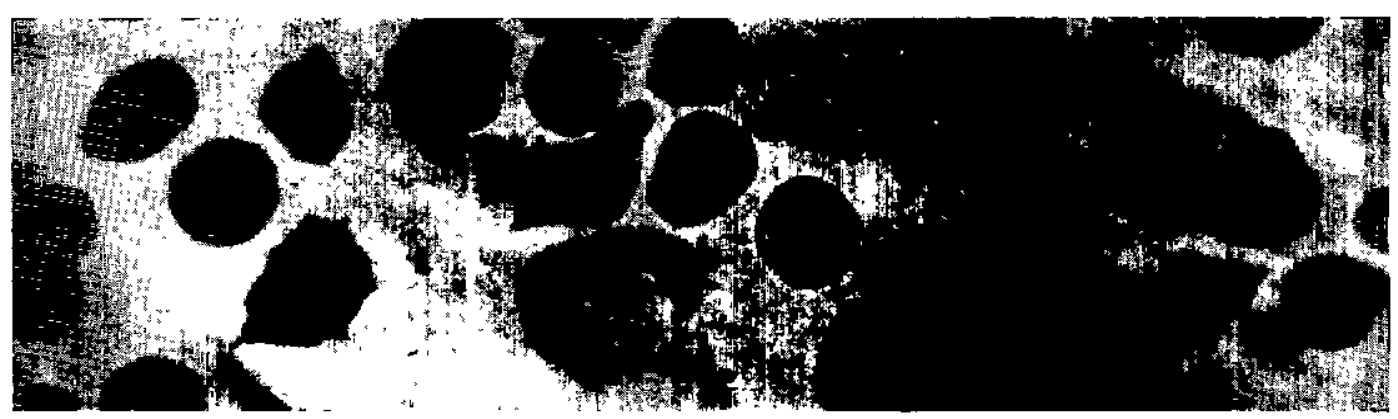

Fig. 6 


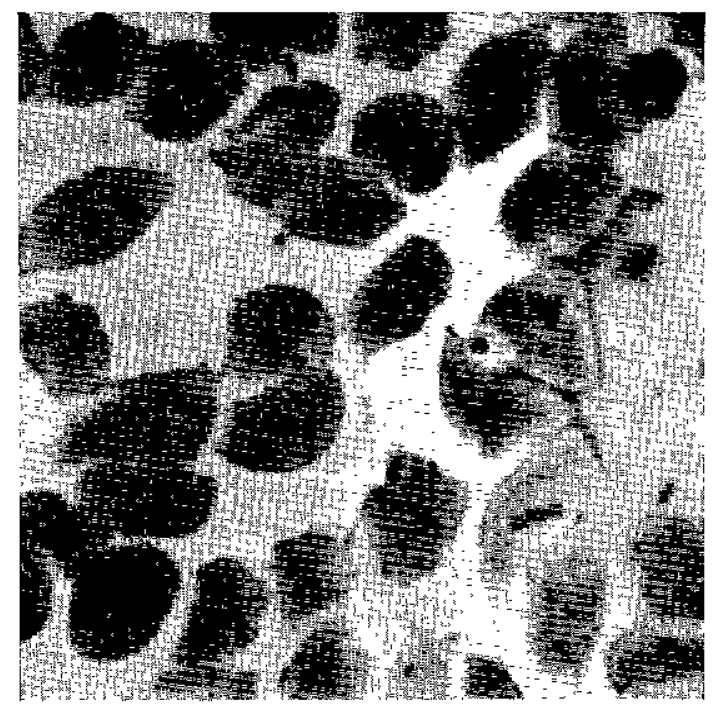

Fig. 7

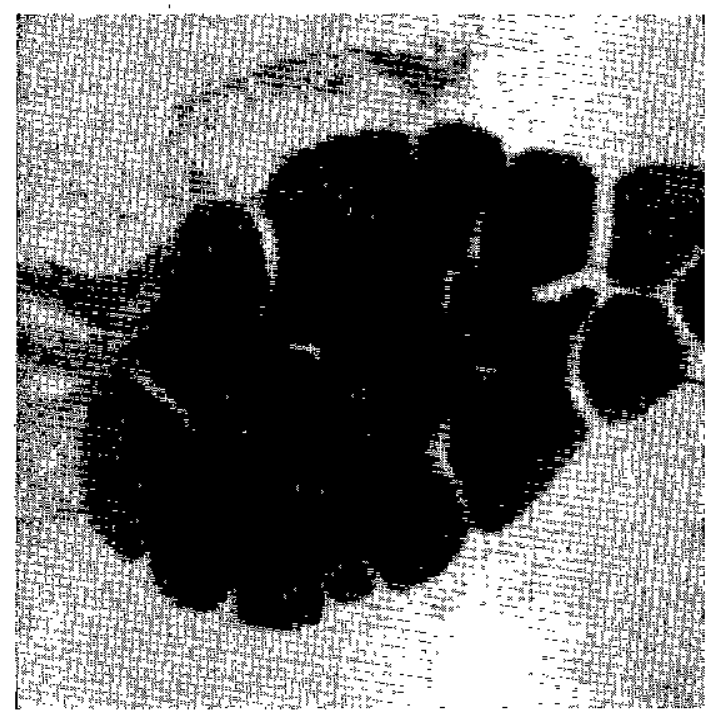

Fig. 9

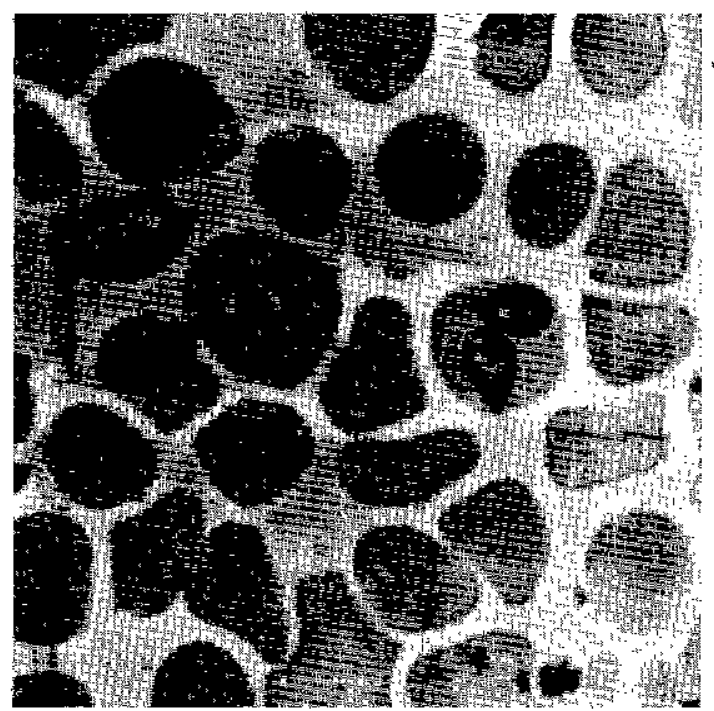

Fig. 11

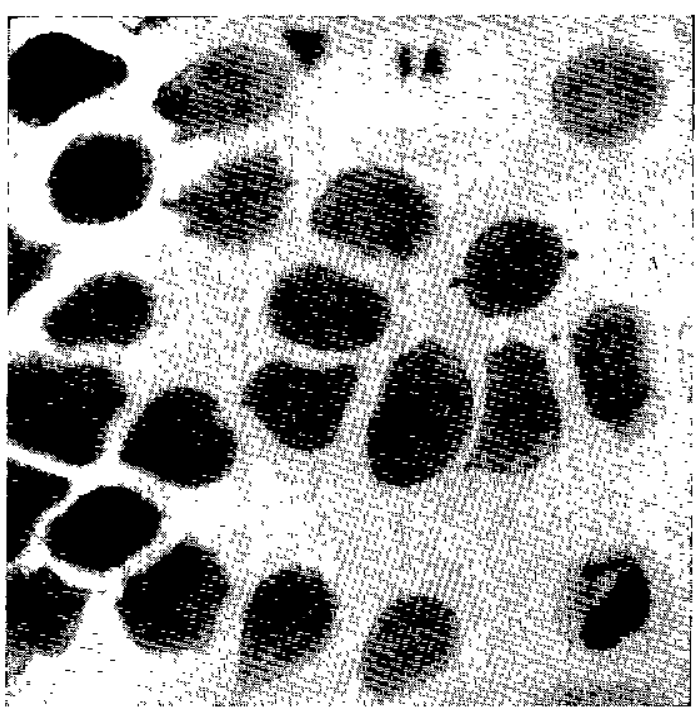

Fig. 8

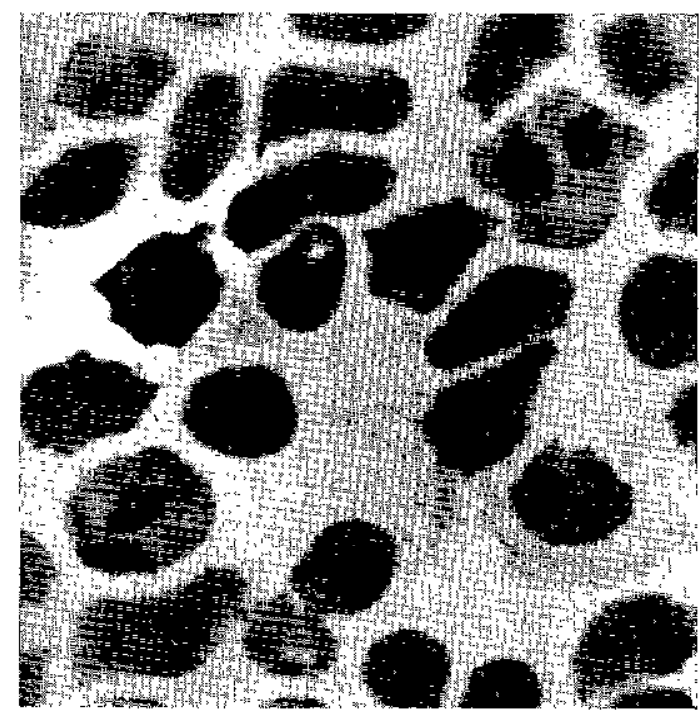

Fig. 10

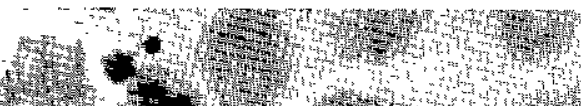

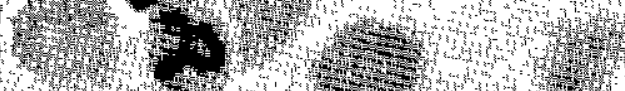

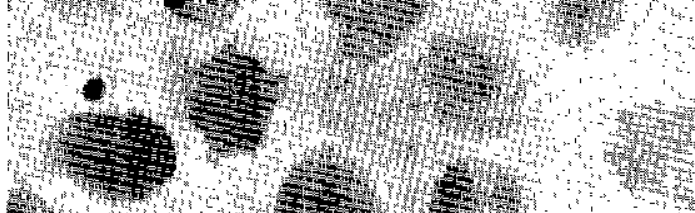

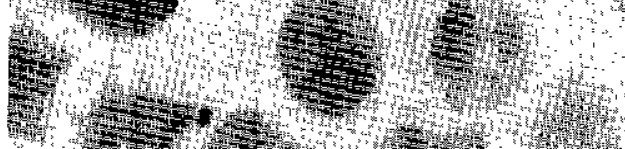

W

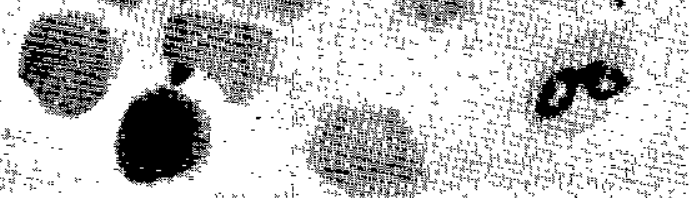

Fig. 12 


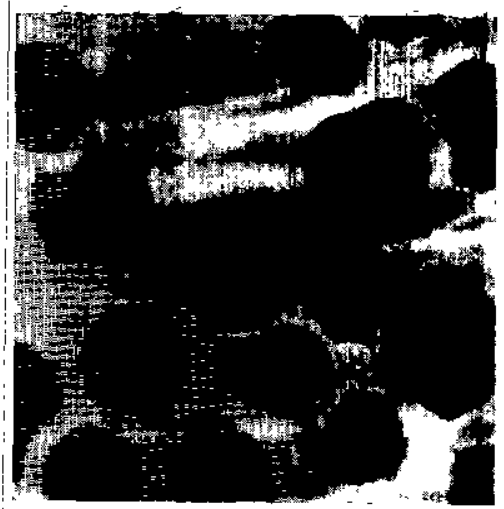

Fig 13

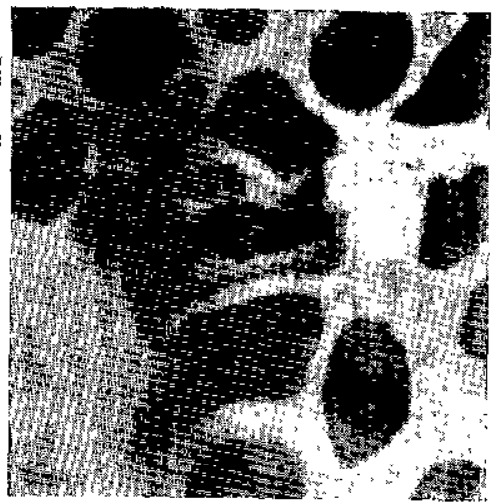

Fig. 16

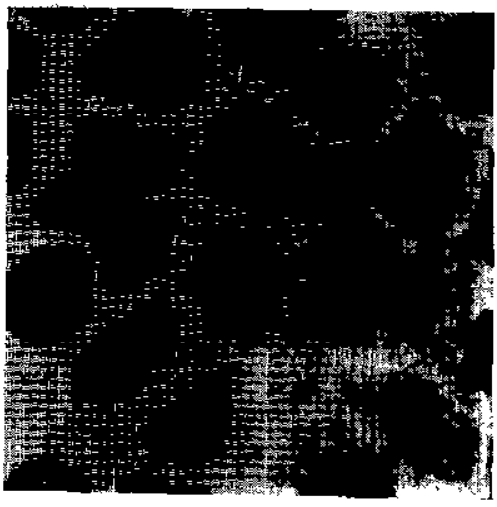

Fig. 19

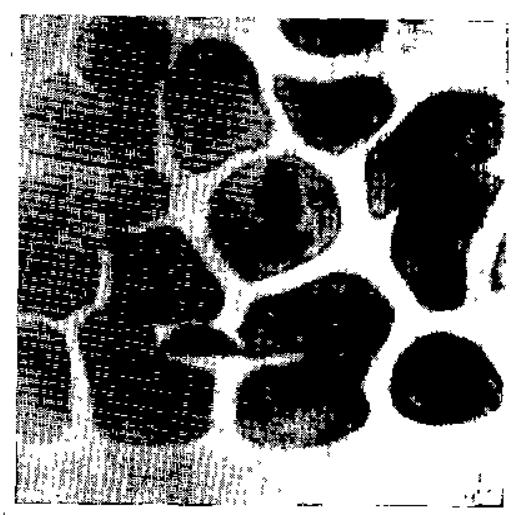

[i] 72

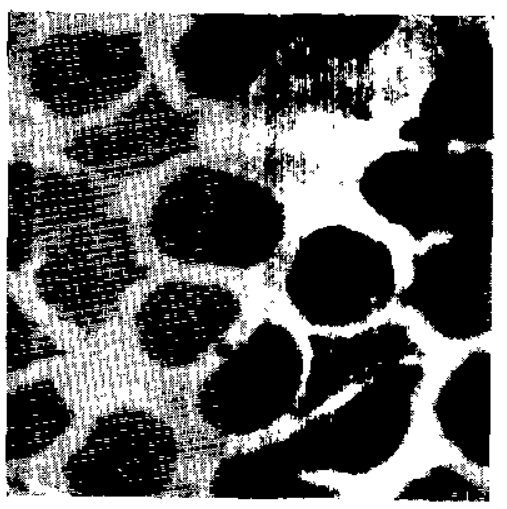

Fig. 14
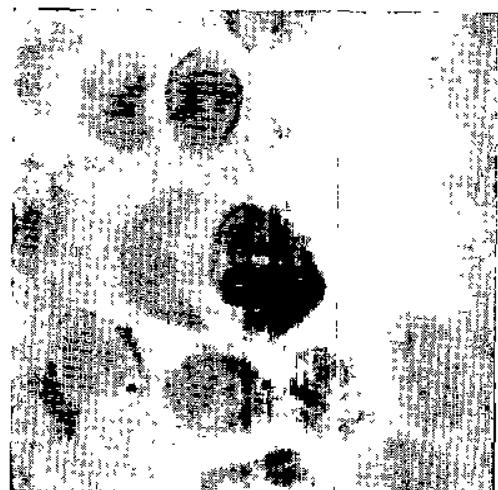

Fig. 17

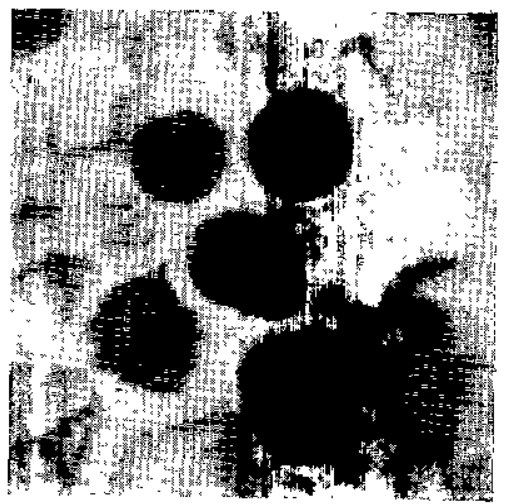

Fig. 20

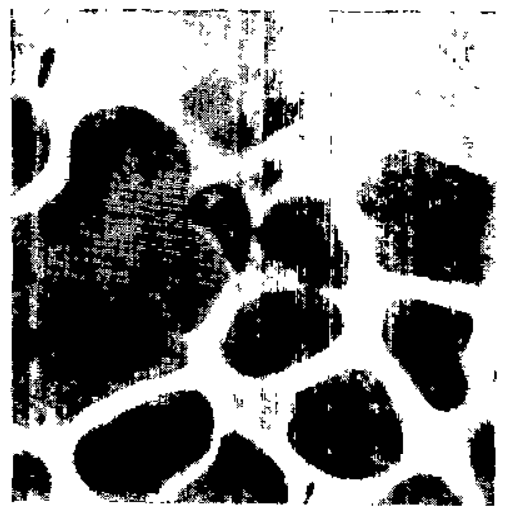

Fig. 23

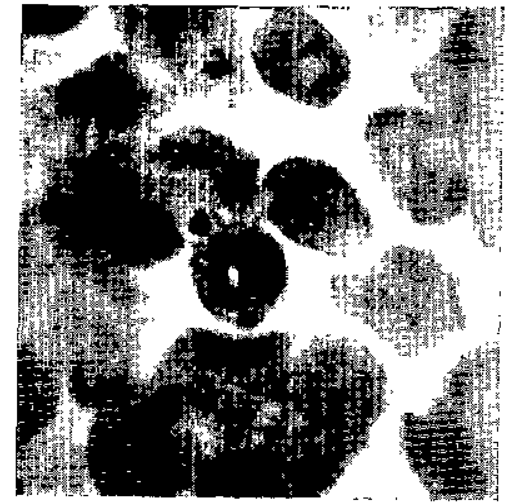

Fig. 15

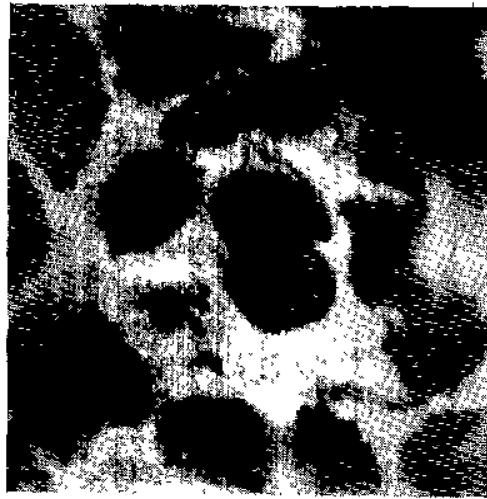

Fig 18

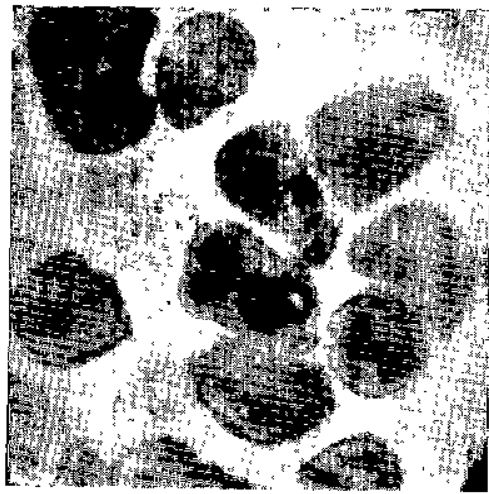

Fig. 21
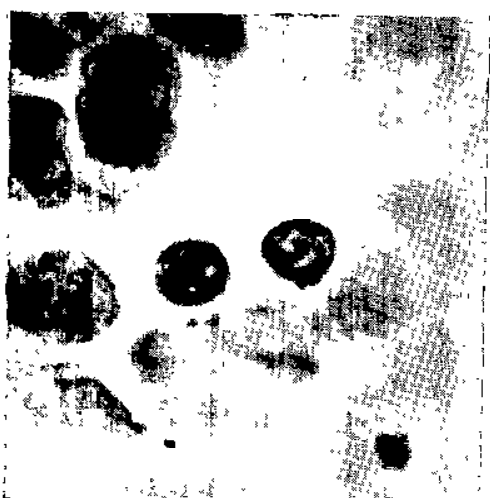

Fig. 24 


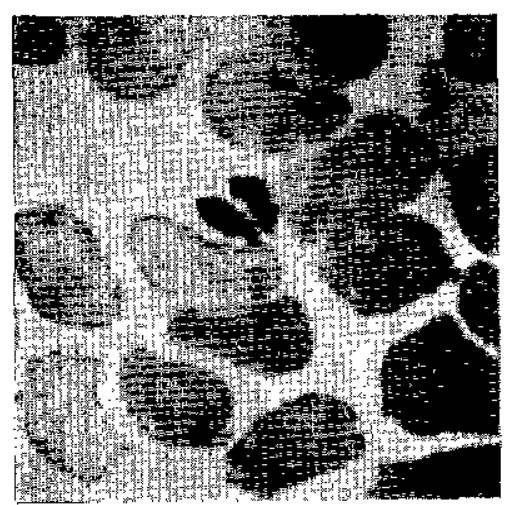

Fig. 25

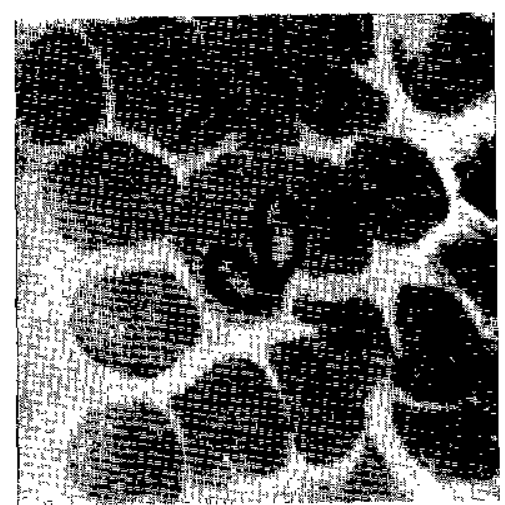

Fig. 28

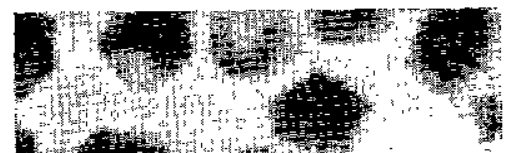
10.1. 1 (2) - 40 Fig. 31

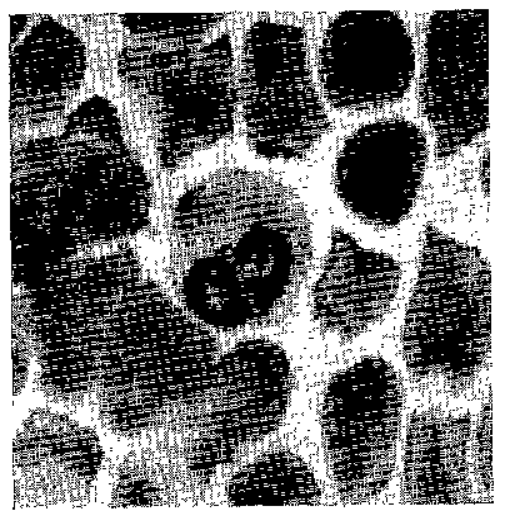

Fig. 34

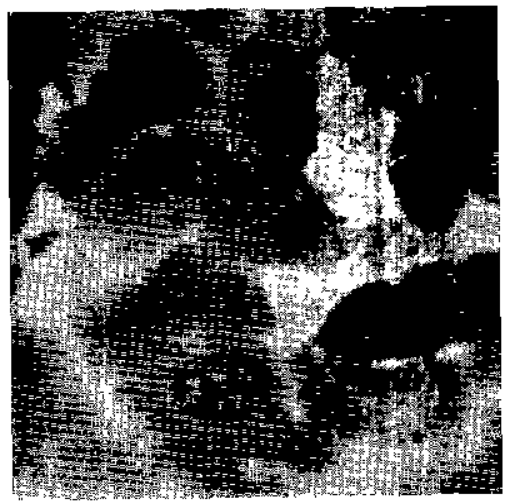

Fig. 26

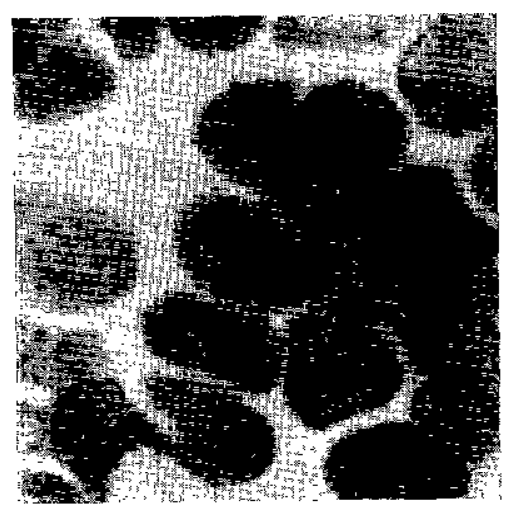

Fig. 29

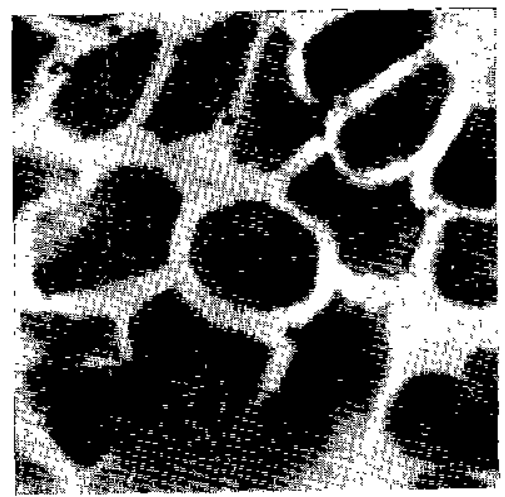

Fig. 32

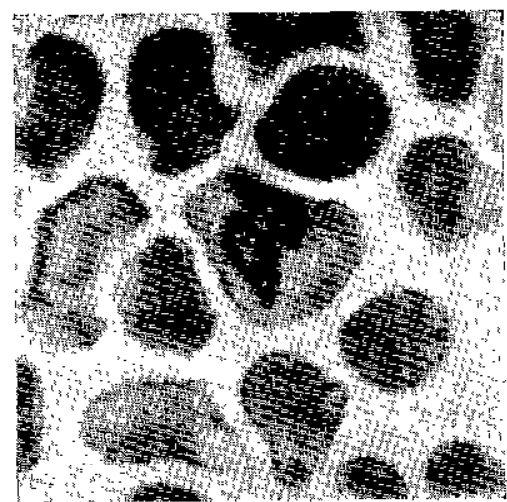

Fig. 35

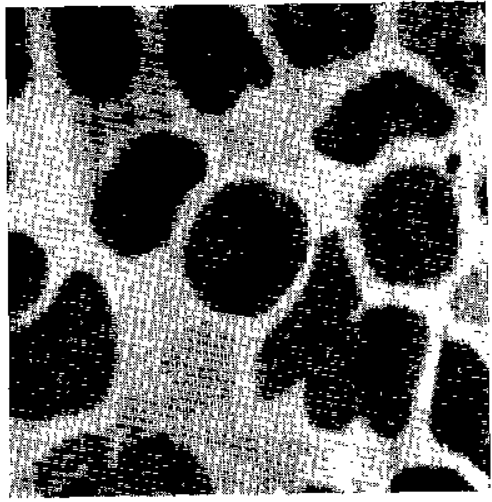

Fig. 27

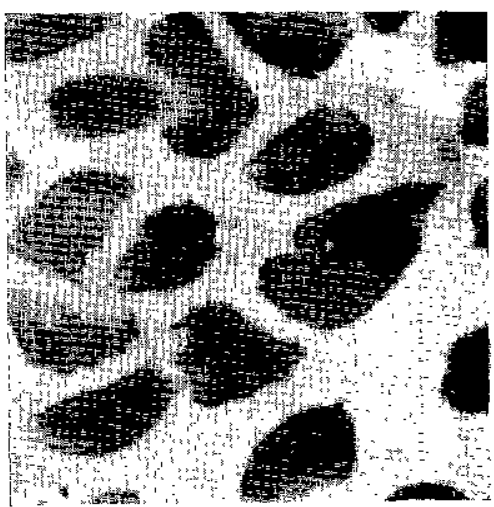

Fig. 30

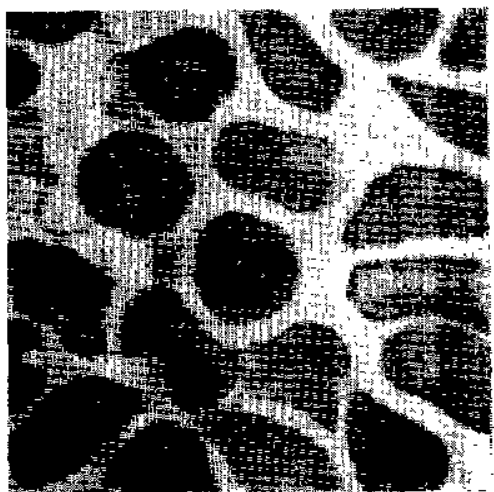

Fig. 33

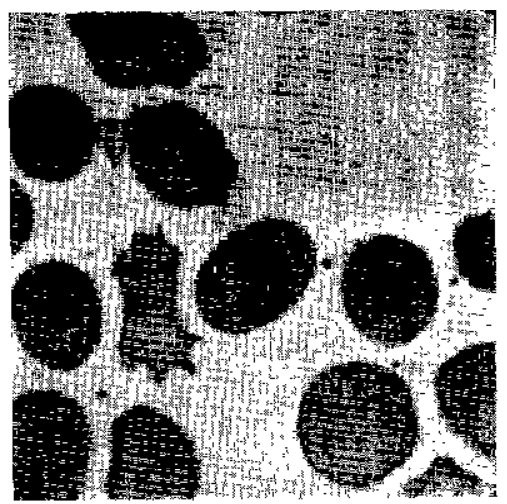

Fig. 36 


\title{
RESUMEN \\ La Piroplasmosis del puerco. \\ Infección natural cón Piroplosma-trautmanni. \\ (KNUTi, Y DU TOIT, 19:) \\ en Bambari (Republica centroafricana)
}

\begin{abstract}
Se observa una infección con Piroplasima trautmann en uná márana curada por una metritis. Los ixodos recogidos pertenecen a la especie Rhipicepholus senegalensıs. Luego de un estudío morfológico del parás|to, se presenta una serie de microfolografias,
\end{abstract}

\section{BIBLIOGRAPHIE}

CERNAIANU (C. C.). - Piroplasme si Piroplasmose, 1958, Vol. II, Academiei Républicii

: Populare Romıne ed., Bucarest.

CERRUTI (C. G.). - Recherches sur les Piroplasmoses du Porc, Ann. Parasit. Hum. Comp., 1939, 17 (2), 114-36.

F. A. O. - Rapport de la deuxième réunion du groupe d'experts F.A.O/O.I.E.surles maladies du bétail transmises par les tiques. Le Caire (R. A. U.) 3-10 décembre 1962. Organisation des Nations Unies pour l'alimentation et I'Agriculture, Rome (Italie).

HOOGSTRAAL (H.). - African, Ixodoidea I Tlaks of the Sudan, 1956. Research Report NM 005.050.29.07.U.5. Government Printing Office : 315.

1. E. M. V. T. - Région de Recherches Vétérinaires et Zootechniques de l'Áfríque Centrale (Laboratoire, de Farcha, Fort-Lamy, Tchad), Rapport annuel $1959: 175$.

JUSSIANT, - Notes cliniques sur quelques ma-

$\therefore$ ladies du bétail. La piroplasmose du porc au Congo belge, Bull. Agrl. Congo Belge. 1948, 39 (3) : 631.

LAWRENCE (D. A.) ef SHONE (D. K.). - Piroplasmose du porc. Infection à Babesia trautmanni en Rhodésie du Sud (Porcine Piroplasmosis Babesia trautmonni infection in Southern Rhodesia). J. S. Afr. Vet. Med. Ass., 1955, 26 (2) : 89-93.

MOREL (P. C.) ef FINELLE (P). - Les tiques des animaux domestiques du Centrafrigue.
Rev. El. Méd. Vét. Pays trop., 1961, 14 (2) : 191-7.

NEITZ (W. O.). - Classification transmission et biologie des Piroplasmes des animaux domestiques (Classification, transmission and biology of Piroplasms of domestic animals). Ann. New York Acad. Sci., 1956, 64 : 56-111. PAVLOV (P.) et PASCHEV (II.). - Recherches sur la Piroplasmose du Porc en Bulgarie. Ann. Parosit. Hum. Comp. 1946, 21 (56): 235-40.

RAYNAUD (J. P.). - Morphologie, chimiosensibilité ef réactions immunitaires de souches de Babesio bigemina (Smith ef Kilborne 1893) mise en évidence par splénectomie de bovins. Rev. Elev. Méd. Vét. Poys trop., 1962, 15 (2) : 167-79.

RICHARDSON (U. F.) et KENDALL. (S. B.). Veterinary Protozoology. Oliver and Boyd ed. Londres, $1957: 150$.

ROUSSELOT (R.). - Notes de Parasitologie tropicale. T. 1., 1953, Vigot, Paris.

SERGENT (E.), DONATIEN (A.), PARROT (L.) et LESTOQUARD (F.). - Etudes sur les Piroplasmoses bovines Institut Pasteur d'Al. gérie, Alger, 1945, 816 pp.

SHONE (D. F.) ef PHILIP (J. R.). - - The susceptibility of the africain bush pig. Potar mochoersș. porcus moschono, Lonnberg, to infection with Babesia trautmanni. J. S. Afr. Vet. Med. Ass, 1960..31 (4) : 451-3.

TEINDERO (J.). - Infestao natural do porco da guine pela Babesia troutmanni Bol. Cult. Guinée portug. 1952, 7 (26) : 359-64. 